\title{
Surgical lung biopsy for interstitial lung disease: when considered necessary, should these be done in larger and experienced centres only?
}

\author{
John Hutchinson (1) ${ }^{1}$, Richard Hubbard ${ }^{2}$ and Ganesh Raghu ${ }^{3}$ \\ Affiliations: 'Sherwood Forest Hospitals, Sutton-in-Ashfield, UK. ${ }^{2}$ University of Nottingham, Nottingham, UK. \\ ${ }^{3}$ University of Washington, Seattle, WA, USA.
}

Correspondence: John Hutchinson, Dept of Respiratory Medicine, King's Mill Hospital, Sutton-in-Ashfield, Nottinghamshire, NG17 4JL, UK. E-mail: johnhutchAdoctors.org.uk

@ERSpublications

Surgical lung biopsy for ILD: higher volume centres may offer lower mortality, but data are only suggestive - we need more research, including into lower risk alternatives to conventional biopsy, to reduce risk for patients needing diagnostic confirmation http://ow.ly/OeAe30nrdMr

Cite this article as: Hutchinson J, Hubbard R, Raghu G. Surgical lung biopsy for interstitial lung disease: when considered necessary, should these be done in larger and experienced centres only? Eur Respir J 2019; 53: 1900023 [https://doi.org/10.1183/13993003.00023-2019].

Ascertaining an accurate diagnosis for people confronted with new onset interstitial lung disease (ILD) is central to informing discussions around prognosis and treatment choices. In people with idiopathic pulmonary fibrosis (IPF), treatment with both pirfenidone and nintedanib has been shown to reduce the rate of disease progression $[1,2]$, whereas historical use of immunosuppression was shown in the landmark PANTHER study to be harmful [3]. In other conditions, such as hypersensitivity pneumonitis or sarcoidosis, an accurate diagnosis helps to inform prognosis, allow removal from harmful exposure, and justify consideration of treatment with corticosteroids and/or immune modulating agents, if required $[4,5]$.

With the advent of high-resolution computed tomography (HRCT) scans of the chest and recognised patterns and distribution of images, an accurate diagnosis can be ascertained in the appropriate clinical setting. For example, in IPF, a definite usual interstitial pneumonia (UIP) pattern on HRCT in the appropriate clinical context (aged over 60 years, bibasal crackles, no alternative exposure history attributable to the manifested ILD or serological markers for connective tissues disease) is sufficient to consider treatment according to latest guidelines [6-8]. However, in the absence of connective tissue diseases and/or exposures of environmental factors known to induce hypersensitivity pneumonitis, and when the HRCT imaging features reveal patterns that are indeterminate for UIP or otherwise, histopathology features, usually by means of a surgical lung biopsy, may be required to make an accurate diagnosis.

This would suggest that a surgical lung biopsy features frequently in the diagnostic work-up for ILD, but this is not necessarily the case [9]. Rates of histological confirmation for IPF vary significantly in epidemiological studies [10-12] and clinical trials $[1,2,13]$. Besides the risks associated with a surgical lung biopsy, concerns about inter-observer variation in interpretation of the histopathology [14], varying pathology in different areas of the lung [15] and the possibility of no clear diagnosis (or unclassifiable disease) despite a surgical lung biopsy are some reasons for the reluctance of some clinicians and patients to opt for surgery. The emerging evidence that a surgical lung biopsy carries a risk of mortality has further 
tipped the scales against subjecting patients to the surgery for some clinicians. Numerous studies, mainly case series, have reported the risks of a surgical lung biopsy in ILD: early single-centre series touted alarming risks, but often included patients who would not be biopsied today with significant pre-morbid risk factors [16], while more recent reviews, albeit heterogenous in their study composition, report 30-day mortality of around $2 \%[17,18]$. A $2 \%$ chance of dying from a diagnostic test, however, suggests it is crucial we learn more about how to ensure surgical biopsy for ILD is as safe as possible.

The study from Fisher et al. [19], published in this issue of the European Respiratory Journal, contributes to this debate. The authors use a large administrative clinical dataset from Ontario, Canada, with over 3000 surgical lung biopsies for ILD over a 13-year period (until 2014). Their primary objective was to consider whether centres that performed higher numbers of surgical lung biopsies had better mortality rates than lower volume centres. There is good reason for this hypothesis: similar data exists for multiple types of surgery $[20,21]$ and sub-specialist surgery such as lung transplantation is usually performed in fewer centres to maintain expertise. Centres with greater procedural experience of surgical lung biopsy might offer more specialist assessment and patient selection, increased surgical skill, or more experienced postoperative care, and confirming this might have implications for where patients choose to undergo surgery.

What do the data show? Firstly, Fisher et al. [19] report a 30 -day mortality of around $2 \%$ for elective procedures. This is comparable to data from the USA and UK $[22,23]$, and suggests we now have an international consensus around the mortality risk for all-comers undergoing planned surgical biopsy for ILD. The mortality from non-elective procedures, however, was far higher at $20 \%$, again similar to other data [22]. This reinforces the need for a debate as to whether there is any place for unplanned biopsies in these high-risk populations. Re-admission within 30 days (possibly suggestive of post-operative complications) occurred in around $10 \%$ of patients in this study. Surgical lung biopsy clearly has a risk profile that needs careful communication with patients.

Secondly, the headline figures suggest a lower post-operative mortality in higher volume centres, as hypothesised. The authors elegantly state that for every additional 50 biopsies performed in a year, the risk of dying within 30 days reduces by $16 \%$. However, when stratifying by elective or non-elective procedures, this effect appears to be driven primarily by urgent and emergency cases, with most variability due to patient characteristics rather than hospital differences. The suggestion of a trend persisted for elective biopsies but was no longer statistically significant.

Finally, the authors noted a surprising increase in post-operative mortality in later years, and they speculate that this may be due to more aggressive biopsy strategies to confirm IPF as a result of recent developments in the field, for example licensing of anti-fibrotics and the risks of immunosuppression. This is slightly concerning and warrants further study in alternative data sources, ideally with more clinical data on underlying risk factors and precise diagnoses to compare fitness of patients being referred for biopsy and whether this has changed over time.

There are several strengths to this work. The authors have used a comprehensive and clinically rich data source covering a population of 13 million people, with appropriate exclusions to ensure no patients with cancer slipped into the mix. They have applied sophisticated modelling to consider the impact of centre volume, with sensible stratification by elective and non-elective patients on the basis of previous work. They were able to assess risk factors identified previously, for example gender and age, as well as the impact of long-term oxygen therapy, which perhaps unsurprisingly was significantly associated with mortality in elective patients. Their results show consistency with studies derived from other datasets in the UK and USA. A major limitation to the study was the lack of adequate power when stratifying by elective versus non-elective procedures, which may explain why the association loses significance for elective cases.

Should clinicians then seek out larger centres when contemplating surgical lung biopsy? The data presented suggest this is primarily an issue for non-elective cases, but the mortality rates are so high for these patients that a major question is whether we should contemplate doing these procedures at all. One explanation for the lower mortality in higher volume centres is that there is more clinical experience and that the clinical care is better in these centres, but it is also possible that the busier centres may know better when to say "no" to a high-risk biopsy, whereas smaller centres may "have a go", less aware of the presence of higher risk features. For elective cases, while the theory is intuitive, the data currently available do not support a major difference between high- and low-volume centres, and the key factor affecting mortality is likely to be optimal patient selection, with a focus on identified risk factors including age, gender and comorbidity. A pre-existing oxygen requirement would suggest even an elective biopsy is overly risky. The suggestion of a trend towards lower mortality for elective patients in higher volume centres justifies further research in this area, however, and a larger study of mortality-volume associations for elective cases, ideally with additional clinical details, would be desirable. Given that the mortality of surgical lung biopsy is comparable to lobectomy for lung cancer [24], a curative procedure, the prospect of 
concentrating expertise and therefore reducing mortality may be a means to lowering procedural risk to a level more acceptable to patients. Nevertheless, the risks associated with non-elective a surgical lung biopsy in the patient with more advanced disease (as indicated by the need for supplemental oxygen at rest) is so substantial that both the confronted clinician and patient should carefully weigh the risk-benefit ratio before subjecting the patient to a surgical biopsy, even in higher volume centres.

Clinicians should also consider whether there are alternatives to surgical lung biopsy in people with undiagnosed ILD. Experts differ in their recommendations on the use of a surgical lung biopsy in certain situations. For example, the recent American Thoracic Society, European Respiratory Society, Japanese Respiratory Society, and Latin American Thoracic Society guidelines make a conditional recommendation for surgical lung biopsy when HRCT shows "probable UIP” [6], whereas a recent Fleischer statement opts against biopsy in this cohort [7]. A growing number of centres are building experience with transbronchial cryobiopsy [25]: this appears to offer good quality samples, a diagnosis in $80 \%$ of cases, and complication rates that are improving with greater understanding of optimal procedural techniques, for example haemorrhage control [26-28]; however, the lack of clear standardisation means that latest guidelines currently make no recommendation for the procedure in indeterminate cases, suggesting that experienced centres work towards a standardised approach before widespread adoption [6]. Work using machine learning and RNA sequencing to identify UIP on conventional transbronchial lung biopsy samples has also shown promise [29-31], and a recent validation of this approach on a prospective cohort may allow a diagnosis without the need to proceed to conventional a surgical lung biopsy [32]. Biomarkers to distinguish subtypes of ILD have been enthusiastically pursued but have not progressed beyond the research sphere so far [6,33-35]. A pragmatic approach to diagnosis would be to loosen criteria for anti-fibrotics (or undertake trials to support this approach), and accept trials of treatment for probable and indeterminate disease on imaging. It is likely that variation exists between multidisciplinary teams on their approach to biopsy, and consensus views on challenging cases would be helpful.

In summary, the study by Fisher et al. [19] identifies an important clinical question with potential significant implications for ILD care. The theory that higher volume centres may yield lower mortality after surgical lung biopsy is attractive, although for elective cases, which should form the vast majority of those biopsied, the data are suggestive rather than conclusive. We need further studies, using either administrative data or potentially prospective registries, to explore this issue further. Such data, building on the current work, could make surgical biopsy a safer and more attractive proposition to people with new onset ILD. At the same time, we need continued research into less invasive methods of diagnosis, such as transbronchial cryobiopsy and molecular classification using machine learning in conventional transbronchial lung biopsy. Ascertainment of an accurate diagnosis with minimum procedure-associated risks is vital to ensure our patients get the subsequent high level of evidence-based care they deserve.

Conflict of interest: J. Hutchinson has nothing to disclose. R. Hubbard has nothing to disclose. G. Raghu reports personal fees from and consultancy work on IPF studies for Boehringer Ingelheim, consultancy work on IPF studies for BMS, Bellerophan, Fibrogen, Gilead, Nitto, Promedior, Roche, Sanofi, Veracyte, Biogen, Genentech, and consultancy work on IPF studies and data and safety monitoring board work for Avalyn, and grants from NIH, outside the submitted work.

\section{References}

$1 \quad$ King TE Jr, Bradford WZ, Castro-Bernardini S, et al. A phase 3 trial of pirfenidone in patients with idiopathic pulmonary fibrosis. N Engl J Med 2014; 370: 2083-2092.

2 Richeldi L, du Bois RM, Raghu G, et al. Efficacy and safety of nintedanib in idiopathic pulmonary fibrosis. $N$ Engl J Med 2014; 370: 2071-2082.

3 Raghu G, Anstrom KJ, King TE Jr, et al. Prednisone, azathioprine, and N-acetylcysteine for pulmonary fibrosis. N Engl J Med 2012; 366: 1968-1977.

4 Salisbury ML, Myers JL, Belloli EA, et al. Diagnosis and treatment of fibrotic hypersensitivity pneumonia. Where we stand and where we need to go. Am J Respir Crit Care Med 2017; 196: 690-699.

5 Spagnolo P, Rossi G, Trisolini R, et al. Pulmonary sarcoidosis. Lancet Respir Med 2018; 6: 389-402.

6 Raghu G, Remy-Jardin M, Myers JL, et al. Diagnosis of idiopathic pulmonary fibrosis. An Official ATS/ERS/JRS/ ALAT Clinical Practice Guideline. Am J Respir Crit Care Med 2018; 198: e44-e68.

7 Lynch DA, Sverzellati N, Travis WD, et al. Diagnostic criteria for idiopathic pulmonary fibrosis: a Fleischner Society White Paper. Lancet Respir Med 2018; 6: 138-153.

8 Raghu G, Rochwerg B, Zhang Y, et al. An Official ATS/ERS/JRS/ALAT Clinical Practice Guideline: treatment of idiopathic pulmonary fibrosis. an update of the 2011 Clinical Practice Guideline. Am J Respir Crit Care Med 2015; 192: e3-19.

9 Cottin V. Lung biopsy in interstitial lung disease: balancing the risk of surgery and diagnostic uncertainty. Eur Respir J 2016; 48: 1274-1277.

10 Ferrara G, Carlson L, Palm A, et al. Idiopathic pulmonary fibrosis in Sweden: report from the first year of activity of the Swedish IPF-Registry. Eur Clin Respir J 2016; 3: 31090.

11 Fernandez Perez ER, Daniels CE, Schroeder DR, et al. Incidence, prevalence, and clinical course of idiopathic pulmonary fibrosis: a population-based study. Chest 2010; 137: 129-137. 
12 Natsuizaka M, Chiba H, Kuronuma K, et al. Epidemiologic survey of Japanese patients with idiopathic pulmonary fibrosis and investigation of ethnic differences. Am J Respir Crit Care Med 2014; 190: 773-779.

13 Noble PW, Albera C, Bradford WZ, et al. Pirfenidone in patients with idiopathic pulmonary fibrosis (CAPACITY): two randomised trials. Lancet 2011; 377: 1760-1769.

14 Nicholson AG, Fulford LG, Colby TV, et al. The relationship between individual histologic features and disease progression in idiopathic pulmonary fibrosis. Am J Respir Crit Care Med 2002; 166: 173-177.

15 Monaghan H, Wells AU, Colby TV, et al. Prognostic implications of histologic patterns in multiple surgical lung biopsies from patients with idiopathic interstitial pneumonias. Chest 2004; 125: 522-526.

16 Utz JP, Ryu JH, Douglas WW, et al. High short-term mortality following lung biopsy for usual interstitial pneumonia. Eur Respir J 2001; 17: 175-179.

17 Han Q, Luo Q, Xie JX, et al. Diagnostic yield and postoperative mortality associated with surgical lung biopsy for evaluation of interstitial lung diseases: a systematic review and meta-analysis. J Thorac Cardiovasc Surg 2015; 149: 1394-1401.

18 Nguyen W, Meyer KC. Surgical lung biopsy for the diagnosis of interstitial lung disease: a review of the literature and recommendations for optimizing safety and efficacy. Sarcoidosis Vasc Diffuse Lung Dis 2013; 30: 3-16.

19 Fisher JH, Shapera S, To T, et al. Procedure volume and mortality after surgical lung biopsy in interstitial lung disease. Eur Respir J 2019; 53: 1801164.

20 Reames BN, Ghaferi AA, Birkmeyer JD, et al. Hospital volume and operative mortality in the modern era. Ann Surg 2014; 260: 244-251.

21 Al-Sahaf M, Lim E. The association between surgical volume, survival and quality of care. J Thorac Dis 2015; 7: Suppl. 2, S152-S155.

22 Hutchinson JP, Fogarty AW, McKeever TM, et al. In-hospital mortality after surgical lung biopsy for interstitial lung disease in the United States. 2000 to 2011. Am J Respir Crit Care Med 2016; 193: 1161-1167.

23 Hutchinson JP, McKeever TM, Fogarty AW, et al. Surgical lung biopsy for the diagnosis of interstitial lung disease in England: 1997-2008. Eur Respir J 2016; 48: 1453-1461.

24 Powell HA, Tata LJ, Baldwin DR, et al. Early mortality after surgical resection for lung cancer: an analysis of the English National Lung cancer audit. Thorax 2013; 68: 826-834.

25 Johannson KA, Marcoux VS, Ronksley PE, et al. Diagnostic yield and complications of transbronchial lung cryobiopsy for interstitial lung disease. A systematic review and metaanalysis. Ann Am Thorac Soc 2016; 13: $1828-1838$.

26 Colella S, Haentschel M, Shah P, et al. Transbronchial lung cryobiopsy in interstitial lung diseases: best practice. Respiration 2018; 95: 383-391.

27 Hetzel J, Maldonado F, Ravaglia C, et al. Transbronchial cryobiopsies for the diagnosis of diffuse parenchymal lung diseases: expert statement from the Cryobiopsy Working Group on Safety and Utility and a call for standardization of the procedure. Respiration 2018; 95: 188-200.

28 Lentz RJ, Argento AC, Colby TV, et al. Transbronchial cryobiopsy for diffuse parenchymal lung disease: a state-of-the-art review of procedural techniques, current evidence, and future challenges. J Thorac Dis 2017; 9: 2186-2203.

29 Pankratz DG, Choi Y, Imtiaz U, et al. Usual interstitial pneumonia can be detected in transbronchial biopsies using machine learning. Ann Am Thorac Soc 2017; 14: 1646-1654.

30 Choi Y, Liu TT, Pankratz DG, et al. Identification of usual interstitial pneumonia pattern using RNA-Seq and machine learning: challenges and solutions. BMC Genomics 2018; 19: Suppl. 2, 101.

31 Kim SY, Diggans J, Pankratz D, et al. Classification of usual interstitial pneumonia in patients with interstitial lung disease: assessment of a machine learning approach using high-dimensional transcriptional data. Lancet Respir Med 2015; 3: 473-482

32 Raghu G, Flaherty K, Lederer D, et al. Usual interstitial pneumonia: prospective validation of a molecular classifier in conventional transbronchial lung biopsy for the diagnosis of UIP. Lancet Respir Med 2019; in press.

33 Guiot J, Moermans C, Henket M, et al. Blood biomarkers in idiopathic pulmonary fibrosis. Lung 2017; 195: 273-280.

34 Jenkins RG, Simpson JK, Saini G, et al. Longitudinal change in collagen degradation biomarkers in idiopathic pulmonary fibrosis: an analysis from the prospective, multicentre PROFILE study. Lancet Respir Med 2015; 3: $462-472$.

35 Raghu G, Richeldi L, Jagerschmidt A, et al. Idiopathic pulmonary fibrosis: prospective, case-controlled study of natural history and circulating biomarkers. Chest 2018; 154: 1359-1370. 